\title{
Laser Therapy Versus Electromagnetic Field on Mucosal Membrane Thickening in Children With Chronic Rhinosinusitis
}

\author{
Nesrin Afify Abdulrashid ${ }^{1}$, Hamada El-Sayed Ayoub ${ }^{2 *}$, Ahmed Mamdouh AbdelKader ${ }^{3}$ \\ 'Department of Physical Therapy for Surgery, Faculty of Physical Therapy, Cairo University, Egypt \\ ${ }^{2}$ Department for Pediatric Physical Therapy, Faculty of Physical Therapy, Cairo University, Egypt \\ ${ }^{3}$ Integumentary Department, Faculty of Physical Therapy, Deraya University, Egypt
}

\author{
*Correspondence to \\ Hamada El-Sayed Ayoub \\ Lecturer, Pediatric P. T. \\ Department, \\ Cairo University, Egypt. \\ Tel: +201220313532 \\ Email: \\ hamada.ayoub@pt.cu.edu.eg \\ Published online July, 62019
}

\begin{abstract}
Introduction: Rhinosinusitis is a frequent disease in children but may be underdiagnosed, that is to say, several causes of the disease are missed in children. Therefore, it seems essential to provide other modalities to treat chronic health conditions like such cases. This controlled clinical study was conducted to compare the effect of two non-pharmacological, painless, safe modalities; low-level laser therapy (LLLT), with pulsed electromagnetic field (PEMF) on mucosal membrane thickening in children with chronic rhinosinusitis.

Methods: Thirty children with chronic sinusitis participated in this study, their ages ranged from 6 to 13 years old, recruited from the outpatients ENT clinic, Kasr Al Aini teaching hospital. They were classified into 2 groups of equal number; study group A (PEMF group) and study group B (LLLT group). These children were evaluated before the starting and after the end of the study through CT scan full examination (coronal and axial) for all children.

Results: This study showed that there were statistically significant improvements in mucosal membrane thickness in both groups $(P<0.05)$, while there was no statistically significant difference between groups $(P>0.05)$.

Conclusion: From the obtained outcomes it can be concluded that both the laser and electromagnetic field can be used effectively in the management of symptoms in children with chronic rhinosinusitis, while no significant difference between these modalities obtained.

Keywords: Rhinosinusitis; LLLT; PEMF; Mucosal membrane thickness; CT scan.
\end{abstract}

\section{Introduction}

Chronic sinusitis is an inflammatory process in which the paranasal sinuses are involved and persists for more than twelve weeks. Pediatric acute and chronic rhinosinusitis are common pediatric condition averaging six to eight occurrences per year, that $0.5 \%-5 \%$ of these progressing to acute sinusitis. Chronic sinusitis is rarely life-threatening, although serious complication as orbital cellulitis, orbital abscesses, epidural empyemas, subdural empyemas, intracerebral abscesses, meningitis, and cavernous sinus thrombosis can occur due to the proximity to the orbit and cranial cavity. ${ }^{1}$

However rhinosinusitis is not life-threatening, it significantly affects the child's school performance and sleep pattern, in general, the disease has a dramatic impact on the health care system and the national economy. Sinusitis categorized based on the extent of symptoms and anatomic site. Acute sinusitis symptoms last for 4 weeks. Subacute rhinosinusitis has mild to moderate symptoms that define to be 4 to 12 weeks duration. Chronic Rhinosinusitis lasts 12 weeks or more and often has pathophysiology different from that of acute sinusitis. ${ }^{2}$ The efficiency of the osteomeatal complex is mainly critical for sinus health. Ostial obstruction is usually the initial point for sinusitis. It creates a negative pressure in the sinuses causing fluid leakage inside the sinuses that can be easily infected. This causes lining cilia damage and increases the mucus production, thus compromises the mucociliary clearance.

A self-perpetuating cycle establishes which needs to be interrupted for the best possible results. ${ }^{3}$ The "osteomeatal complex" obstruction can be caused by many factors as anatomic abnormalities, mucosal edema, non-allergic rhinitis, unattended nasal foreign bodies, gastroesophageal reflux, smoking either active or passive and environmental pollution and irritants. ${ }^{4,5}$

Please cite this article as follows: Afify Abdulrashid NA, Ayoub HE, AbdelKader AM. Laser therapy versus electromagnetic field on mucosal membrane thickening in children with chronic rhinosinusitis. J Lasers Med Sci. 2019;10(3):230-234. doi:10.15171/jlms.2019.37. 
One or more sinuses may get infected. Isolated sphenoidal sinusitis is less common and detects in only $2.7 \%$ of sinuses infections. ${ }^{4,5}$ The typical cases of sinusitis are medically treated, which can decrease the mucosal inflammation and edema, relieving the pain, struggle the infection, open the ostia of the sinuses and recover normal mucociliary functions. Nevertheless, surgical interference operates when; sinusitis is not medically controlled, fast progressive sinusitis happens, sinusitis causes an abscess either in the sinus or nearby areas as the orbit or brain and sinusitis that compromises the survival of the patient occurs..$^{6-8}$ Low-level laser therapy (LLLT) is a light therapy that being used by physiotherapists to treat a wide range of acute and chronic musculoskeletal disorders, by dentists to treat inflamed oral tissues and to cure different ulcerations, by dermatologists to cure edema, buns and dermatitis, by rheumatologists to alleviate pain and treat chronic inflammation and autoimmune disease, and by other specialists, as well as general practitioner. Also, LLLT has an effect on the immune system, microcirculation, anti-inflammatory, analgesic, and bactericidal effect, so it can be used in rhinosinusitis cases. ${ }^{9}$

The point of using LLLT is to provide direct biostimulation to the body's cells through the energy of the laser. Photoreceptors present in the cells (e.g., cytochromophores and antenna pigments) can take up low-level laser light, carrying it to mitochondria, producing the cells' energy or ATPs. ${ }^{10}$

Pulsed electromagnetic field (PEMF) has an effect on cell behavior by changing the electrical activity around and within the cell. In addition, it could augment oxygen pressure, activate and regenerate cells induced by enhancing blood supply. Moreover, more calcium transfer increases absorption of calcium in bones and improves the cartilaginous integrity in the joints, so alleviating pain considerably. Furthermore, acute and even chronic pain caused by osteoporosis may entirely fade away. Also, the PEMF improve circulation and blood flow which is beneficial in reducing sinus inflammation. ${ }^{11}$ The hypothesis of the study was null; there would have been no difference between LLLT and PEMF on rhinosinusitis in children.

\section{Methods}

\section{Study Design}

The investigation designed as a pre and posttest randomized controlled clinical study. All of the enrolled children were complaining from chronic sinusitis. Before taking the informed consent the entire process used in the study and its investigational character explained to the children parents or caregivers.

\section{Subjects}

The inclusion criteria regarding the thirty children participated in this study; They were suffering from chronic rhinosinusitis as referred by the physician for more than 2 years, they received medical treatment including antihistamines and antibiotics during seasonal attacks in the previous 2 years, both genders were involved, their ages were from 6 to 13 years, and they were free from any immune-deficiency disorders or diseases that might interfere with the healing process and influence the results. They were selected from the Ear, Nose, and Throat (ENT) Department of Kasr El-Aini teaching Hospital in Egypt. They were assigned into 2 groups of equal number; study group A (PEMF group) and study group B (LLLT group).

\section{Treatment Procedure}

The children participated in the study did not receive any medical treatment during the study including antihistaminic or antibiotics. An automatic PMT Quattro PRO used, and it consists of PMT Quattro PRO generator and one couch with $80 \mathrm{~cm}$ solenoid.

An electromechanical movement controlled by a microprocessor featured to position automatically the solenoid.

Each child in group A was placed comfortably in the solenoid of PEMF device, the device was switched on, selecting the appropriate program (20 gausses for 10 minutes, $7 \mathrm{~Hz}$ ), and this protocol of treatment was conducted 3 sessions/week, for 1 month.

The Ga-As (infrared red) laser device consisted of a laser unit which is a small handheld machine class I, laser product under the existing requirement of limited states of food and drug association regulation. It manufactured by Laserex Technologies Pty Ltd., Australia. Each child was instructed to wear the protective eyeglasses while applying the laser to prevent permanent eye damage caused by direct exposure to the beam. Each child in group B placed in a comfortable position, wear the protective eyeglasses, then the device was switched on, the appropriate program was selected (1.5 J for all sinuses for 8 minutes this dose was divided on the eight sinuses, $9.12 \mathrm{~Hz}, 905 \mathrm{~nm}$ ), and this protocol of treatment was conducted 3 sessions/week, for 1 month.

\section{Assessment}

The children were sent to the radiological center for CT scanning examination before the beginning and after the end of the treatment, based on the protocol for detecting mucosal membrane thickness in millimeters $(\mathrm{mm})$.

\section{Statistical Analysis}

Wilcoxon matched pairs test was used to compare the mucosal membrane thickness ( $\mathrm{mm}$ ) within groups (before and after treatment), while the Mann-Whitney test was used to compare the mucosal membrane thickness (mm) between groups.

\section{Results}

This study was conducted to evaluate the efficiency of 
PEMF against LLLT on mucosal membrane thickness in children with chronic rhinosinusitis.

There were 2 independent variables, the first one was (the tested groups) between subjects' factor which had 2 groups; group A received PEMF and group B received LLLT. The second one was (the training period) within subjects' factor which had 2 levels pre and post. In addition, there was one dependent variable (mucosal membrane thickness).

Thirty children suffering from CRS from both genders enrolled in the study, classified into 2 groups; group A (PEMF) consisted of 15 children ( 8 boys and 7 girls), the mean \pm SD for their ages was $6.47 \pm 3.23$. While group B (LLLT) consisted of 15 children (9 boys and 6 girls), the mean \pm SD for their ages was 7.6 \pm 2.97 .

Mucosal Membrane Thickening on the Right Side

i) Within-Subjects

\section{Group A}

Table 1 demonstrated pre- and post-treatment measures of the mucosal membrane $(\mathrm{mm})$ thickening on the right side for the group A. The Wilcoxon matched pairs test revealed a significant reduction of mucosal membrane thickness between pre- and post-treatment values. The median value for pretreatment was 0.5 and post-treatment was 0 , while $P$ value was 0.02 .

\section{Group B}

Table 2 demonstrated the mucosal membrane thickening on right side pre- and post-treatment for the group B. The Wilcoxon matched pairs test revealed a significant reduction of mucosal membrane thickness between pre- and post-treatment values, as the median value of pretreatment was 4 and for post-treatment was 2.7 while $P$ value was 0.01 .

Table 1. Pre- and Post-treatment Median, Range, and $P$ Values of Mucosal Membrane Thickening on the Right Side for Group A

\begin{tabular}{lcc}
\hline \multirow{2}{*}{ Group A } & \multicolumn{2}{c}{ Mucosal Membrane Thickening on Right Side } \\
\cline { 2 - 3 } & Pre-treatment & Post-treatment \\
\hline Median & 0.5 & 0 \\
Range & 8 & 6.2 \\
$Z$-value & & 2.2 \\
$P$ value & $0.02 *$ & \\
\hline
\end{tabular}

*Significant.

Table 2. Median, Range, and P values of Mucosal Membrane Thickening on Right Side Pre and Post-treatment for Group B

\begin{tabular}{lcc}
\hline \multirow{2}{*}{ Group B } & \multicolumn{2}{c}{ Mucosal Membrane Thickening on Right Side } \\
\cline { 2 - 3 } & Pre-treatment & Post-treatment \\
\hline Median & 4 & 2.7 \\
Range & 9 & 7.3 \\
$Z$-value & & 2.36 \\
$P$ value & $0.01^{*}$ \\
\hline
\end{tabular}

\section{ii) Between Groups}

It is demonstrated in Table 3 that, there was no significant difference in the mucosal membrane thickening on the right side before and after treatment between groups $\mathrm{A}$ and $\mathrm{B}$. The $P$ value was 0.2 , and 0.14 respectively.

\section{Mucosal Membrane Thickening on the Left Side}

i) Within-Subjects

\section{Group A}

As shown in Table 4; when comparing the mucosal membrane thickening on the left side before and after treatment for the group A. There was a significant reduction of mucosal membrane thickness, as the median value of pretreatment was 1.5 and for posttreatment was 1.5 while the $P$ value was 0.01 .

\section{Group B}

As demonstrated in Table 5, when comparing the mucosal membrane thickening on the left side before and after treatment for the group B, there was a significant reduction of the membrane thickness, as the median value of pretreatment was 3 and for posttreatment was 2.7 while the $P$ value was 0.04 .

\section{ii) Between Groups}

As shown in Table 6, there was no significant difference in the mucosal membrane thickening on the left side

Table 3. Mucosal Membrane Thickening $(\mathrm{mm})$ on the Right Side Before and After Treatment Between Groups A and B

\begin{tabular}{lcc}
\hline \multirow{2}{*}{$\begin{array}{l}\text { Mann-Whitney } \\
\text { test }\end{array}$} & \multicolumn{1}{c}{ Mucosal Membrane Thickening on Right Side } \\
\cline { 2 - 3 } Pre-treatment & Post-treatment \\
\hline value & 83.0 & 79.5 \\
$P$ value & 0.2 & 0.14 \\
Significance & $\mathrm{NS}$ & $\mathrm{NS}$ \\
\hline NS: non-significant. & &
\end{tabular}

Table 4. Median, Range, and P values of Mucosal Membrane Thickening on Right Side Pre and Post-treatment for Group A

\begin{tabular}{|c|c|c|}
\hline \multirow{2}{*}{ Group A } & \multicolumn{2}{|c|}{ Mucosal Membrane Thickening on Left Side } \\
\hline & Pre-treatment & Post-treatment \\
\hline Median & 1.5 & 1.5 \\
\hline Range & 10 & 7 \\
\hline Z-value & \multicolumn{2}{|c|}{2.37} \\
\hline$P$ value & \multicolumn{2}{|c|}{$0.01 *$} \\
\hline
\end{tabular}

Table 5. Median, Range, and P values of Mucosal Membrane Thickening on Left Side Pre and Post-treatment for Group B

\begin{tabular}{|c|c|c|}
\hline \multirow{2}{*}{ Group B } & \multicolumn{2}{|c|}{ Mucosal Membrane Thickening on Left Side } \\
\hline & Pre-treatment & Post-treatment \\
\hline Median & 3 & 2.7 \\
\hline Range & 10 & 10 \\
\hline Z-value & \multicolumn{2}{|c|}{2.03} \\
\hline$P$ value & \multicolumn{2}{|c|}{$0.04 *$} \\
\hline
\end{tabular}


Table 6. Mucosal Membrane Thickening $(\mathrm{mm})$ on the Left Side Before and After Treatment Between Groups A and B

\begin{tabular}{lcc}
\hline \multirow{2}{*}{$\begin{array}{l}\text { Mann-Whitney } \\
\text { test }\end{array}$} & \multicolumn{2}{c}{ Mucosal Membrane Thickening on Left Side } \\
\cline { 2 - 3 } & Pre-treatment & Post-treatment \\
\hline U value & 97.0 & 107.5 \\
$P$ value & 0.5 & 0.82 \\
Significance & NS & NS \\
\hline
\end{tabular}

NS: non-significant.

before and after treatment between groups $\mathrm{A}$ and $\mathrm{B}$. The $P$ values were 0.5 , and 0.82 respectively.

\section{Discussion}

According to the findings of the current study; the patient in the PEMF group reported that there was an improvement in the mucosal membrane thickness especially the Lt Maxillary sinuses, this may be due to the anti-inflammatory, antimicrobial, analgesic of PEMF, other cellular changes (No, Ca, Production), and also effect of magnetic field on the microcirculation of mucosal membrane of the nasal cavity. ${ }^{12}$

The improvement of membrane thickness may be due to the effect of magnetic therapy on circulation; these results are parallel with Strauch et $\mathrm{al}^{13}$ who confirmed that the magnetic therapy improves tissue inflammation healing through improving blood circulation.

Increased blood flow to the wound or aching area means the increasing supply of nutrients to the affected region, together with the sooner elimination of waste products. In addition, PEMF may have an effect on the iron in the blood (in the form of a charged ion) to be attracted by a magnetic field, that in turn produces heat and increases blood flow. Also, it could be beneficial in reducing mucosal membrane thickness. ${ }^{14}$

The improvement of mucosal membrane thickness may be due to that PEMF that have several impacts on different body systems. Electromagnetic field (EMF) is considered to lengthen the life span of the free radicals and change enzyme activity this comes in agreement with Toshikawa et $\mathrm{al}^{15}$ who reported that; the introduction of nitric oxide synthesis (NOs) occurs when different stresses are present, such as bacterial lipopolysaccharide (LPS) exposure. The administration of the EMF enhances LPS induced NO generation resulting in increasing the free radical lifespan.

According to this study: the patients in the group B (laser group) reported that there was improvement of mucosal membrane thickness especially after the first four sessions of treatment, this might be due to the induced anti-inflammatory and decongesting effect of Ga-As LLLT along with the associated immune effect of laser through restoring the immune-competence cells efficiencies.

Ga-As laser diodes or the infrared laser penetrate more deeply than any other commonly used therapeutic laser. ${ }^{16}$ This phenomenon is largely a result of the fact that the Ga-As diode operates at a wavelength of 904 $\mathrm{nm}$ or $905 \mathrm{~nm}$ and is also due to its super pulsing mode of energy delivery according to the rule that; the higher the wavelength of a therapeutic laser, the deeper the penetration. ${ }^{17}$

The improvement of mucosal membrane thickness may be due to the anti-inflammatory effect of the laser through increasing the phagocyte efficiency, the amount and thickness of lymphatic vessels leads to reducing the permeability of blood vessels and reestablishment of microcapillary circulation; causes edema reduction induced by regaining the normal permeability of vascular wall. ${ }^{18}$

It has believed that LLLT causes biochemical inflammatory markers modulation and induces local anti-inflammatory effects in cells and soft tissue. ${ }^{19}$

Laser therapy has a direct impact on microcirculation and decreases the potential of relapses. Low-energy helium-neon laser modulates vessel permeability, decreases perivascular edema and blood rheology (red blood cell aggregation), and leads to the significant decrease of the mucosal membrane thickness in the children with chronic rhinosinusitis. ${ }^{20}$ Finally, according to the results of the current study, both PEMF and LLLT were effective in improving mucosal membrane thickness in children with rhinosinusitis, while there was no significant difference between PEMF and LLLT in the treatment of chronic sinusitis.

\section{Ethical Considerations}

The protocol of the current study accepted by the ethics committee, Faculty of Physical Therapy, Cairo University (No: P.T.REC/003/001735, 07/03/2017). After a clear explanation of the experimental protocol, the written consents obtained from all participants and their parents or families. This randomized controlled trial was approved by the Pan African Clinical Trial Registry (PACTR), the Cochrane South Africa and registered by the unique identification number of the registry (PACTR201811567176951).

\section{Conflict of Interests}

The authors declare no conflict of interest.

\section{References}

1. Slavin RG, Spector SL, Bernstein IL, et al. The diagnosis and management of sinusitis: a practice parameter update. J Allergy ClinImmunol. 2005;116 (6 Suppl):S13-47.

2. Meltzer EO, Hamilos DL, Hadley JA, et al. Rhinosinusitis: establishing definitions for clinical research and patient care. J Allergy Clin Immunol. 2004;114(6 Suppl):155-212. doi:10.1016/j.otohns.2004.09.067

3. Stevens HE. Allergic and inflammatory aspects of chronic Rhinosinusitis. J Otolaryngol. 1991;20(6):395-399.

4. Ah-See K. Sinusitis (acute). BMJ Clin Evid (Online). 
2011;2011:0511.

5. Orlandi RR, Kennedy DW. Surgical management of Rhinosinusitis. Am J Med Sci. 1998;316(1):29-38. Doi:10.1016/S0002-9629(15)40368-4

6. McQuillan L, Crane LA, Kempe A. Diagnosis and management of acute sinusitis by pediatricians. Pediatrics. 2009;123(2):e193-e198. doi: 10.1542/peds.2008-2479

7. Ferguson BJ. Categorization of eosinophilic chronic rhinosinusitis. Curr Opin Otolaryngol Head Neck Surg. 2004;12(3):237-242.

8. Marple BF, Ryan MW. Allergic fungal rhinosinusitis: diagnosis and management. Curr Opin Otolaryngol Head Neck Surg. 2007;15(1):18-22. doi: 10.1097/ MOO.0b013e328013dbd9

9. Karu T. Biomedical Photonic Handbook. New York: CRC Press; 2003:1-6.

10. Passarella S, Ostuni A, Atlante A, Quagliariello E. Increase in the ADP/ATP exchange in rat liver mitochondria irradiated in vitro by helium-neon laser Biochem Biophys Res Commun. 1988;156:978-86.

11. Vadalà M, Vallelunga A, Palmieri L, Palmieri B, MoralesMedina JC, Iannitti T. Mechanisms and therapeutic applications of electromagnetic therapy in Parkinson's disease. Behav Brain Funct. 2015;11:26. doi: 10.1186/ s12993-015-0070-z.

12. Imants $\mathrm{D}$, Laimdota $\mathrm{D}$, Ilgonis $\mathrm{K}$, Arta $\mathrm{T}$, Bella $\mathrm{A}$, Leonid S. Experimental study of the effect of electromagnetic fields in early stage of wound healing. Bioelectrochem Bioenerg. 1994;35:13-17.

13. Strauch B, Herman C, Dabb R, Ignarro LJ, Pilla AA. Evidence-Based use of pulsed electromagnetic field therapy in clinical plastic surgery, Aesthet Surg J. 2009;29 (2):135-
143. doi: 10.1016/j.asj.2009.02.001

14. Santini MT, Ferrante A, Romano R, et al. A $700 \mathrm{MHz}$ $1 \mathrm{H}-\mathrm{NMR}$ study reveals apoptosis-like behavior in human K562 erythroleukemic cells exposed to a $50 \mathrm{~Hz}$ sinusoidal magnetic field. Int J Radiat Biol. 2005;81(2):97-113. doi: 10.1080/09553000500102985

15. Toshikawa $\mathrm{T}$, Taniqawa $\mathrm{M}$, Taniqawa $\mathrm{T}$, Imai $\mathrm{A}$, Honqo $\mathrm{H}$, Kondo M. Enhancement of nitric oxide generation by low frequency electromagnetic field, Pathophysiology. 2000;7(2):131-135.

16. Tuner J, Hode L. The Laser Therapy Handbook. Sweden: Prima Books; 2004: 43.

17. Kneebone WJ. Practical applications of low level laser therapy. Pract Pain Manag. 2006;6(8):34-40.

18. Lopez RM, Vilchez MA, Gargallo AJ, Arnabat DJ, Gay EC. Efficacy of low level laser therapy in the management of pain facial swelling, and postoperative traismus after a lower third molar extraction, a preliminary study. Lasers Med Sci. 2012;27(3):559-566. doi: 10.1007/s10103-0110936-8.

19. Bjordal JM, Johnson MI, Iversen V, Aimbire F, LopezMartins RA. Low level laser therapy in acute pain: a systematic review of possible mechanism of action and clinical effects in randomized placebo-controlled trials. Photomed Laser Surg. 2006;24(2):158-68. doi: 10.1089/ pho.2006.24.158

20. Kruchinina I, Feniksova LV, Rybalkin SV, Pekli FF. Therapeutic effect of helium-neon laser on microcirculation of nasal mucosa in children with acute and chronic maxillary sinusitis as measured by conjunctival biomicroscopy. Vestn Otorinolaringol. 1991;(3):26-30. 